Case Report

\title{
A RARE EXTRA-PULMONARY PRESENTATION OF TUBERCULOSIS AS GUM TUBERCULOSIS
}

\author{
Srivastava GN ${ }^{1}$, Meena $\mathbf{M}^{2}$, Yadav $\mathrm{P}^{3}$, Hussain $\mathrm{M}^{4}$, Amrita ${ }^{5}$, Kumar $\mathrm{V}^{6}$ \\ 1,2,3,4 Department of TB \& Respiratory Diseases, IMS, BHU, Varanasi, India \\ Department of Pathology, IMS, BHU Varanasi, India \\ Department of General Medicine, IMS, BHU, Varanasi, India
}

\begin{abstract}
Tuberculosis (TB) is a chronic granulamatous disorder presenting mostly (80\%) as a pulmonary disease, the extra-pulmonary presentation being relatively uncommon. The oral cavity can sometimes be a site for extra-pulmonary tuberculosis with a highly variable clinical presentation including ulceration, diffuse inflammatory lesions, granulomas and fissures. Oral lesions usually appear secondary to a primary tubercular infection elsewhere, although primary infection of the oral mucosa by Mycobacterium tuberculosis has been described. We report here a rare case of primary tuberculosis of the hard palate extending to gingiva, manifesting as gingival enlargement with ulceration. Diagnosis was established by histopathological examination of tissue biopsy and by chest radiography. The patient was put on anti-tubercular therapy for nine months to which a prompt response was obtained. This emphasizes the need to consider tuberculosis in the differential diagnosis of non-healing mucosal lesions of oral cavity for early diagnosis and prompt treatment.
\end{abstract}

Key words: Gingiva, Oral Cavity, Tuberculosis

\section{INTRODUCTION}

Tuberculosis is a major public health concern especially in India, and is still among the most life-threatening infectious diseases, resulting in high mortality in adults. With a prevalence of 200 cases per 100000 population in 2009 globally, it is estimated that two billion people (i.e. one-third of the world's population) have been in contact with the tubercle bacillus. The two countries with the largest number of incident cases in 2009 were India and China, India alone accounting for an estimated one fifth $(21 \%)$ of all TB cases worldwide. Moreover, the emergence of drug-resistant TB has recently raised serious concerns. There were an estimated 440000 cases of multi-drug resistant TB (MDR-TB) in 2008, approximately $25 \%$ of them

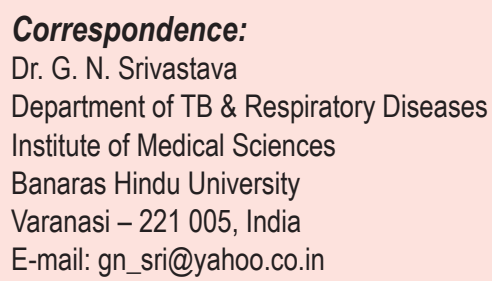

being in India. ${ }^{1}$ Oral TB accounts for $0.1-5 \%$ of all TB infections according to varied reports. ${ }^{2}$ Although oral manifestations of TB have been rare in recent years, they are re-appearing on account of the emergence of the global pandemic of HIV and the rampant drug resistance in tubercle bacilli. ${ }^{3}$ Oral TB accounts for up to $1.33 \%$ of HIV-associated opportunistic infections. ${ }^{4}$ Hence, it is important to consider tuberculosis as a differential diagnosis of oral lesions especially in regions of high incidence of tuberculosis like India. We are presenting here a rare case of primary oral tuberculosis of the hard palate that underlines the importance of the above statement.

\section{Case presentation}

A 26 year old female patient had developed a diffuse, painless, erythematous swelling of the mucosa of hard palate extending anteriorly behind the incisors over a period of 2 years and progressing to ulceration for which she consulted a dentist. She also had systemic complaints of low grade fever, weight loss, loss of appetite. The dentist found no evidence of cervical lymphadenopathy and noted that there was no history of any dental procedures 
or trauma. The patient was treated empirically with antibacterial agents for 1 week with no response. A radiograph of the upper mandible found no bony erosions. She was later put on antifungal therapy and the exudate was sent for potassium hydroxide staining to identify fungal infection but report came back negative. By this time her disease had progressed to involve gingiva and periodontal tissue and 4 upper incisors were unsalvageable and had to be removed. She was then referred to us to rule out tuberculosis. When she presented to us she had an erythematous swelling over hard palate extending to alveolus anteriorly. Rest of the oral cavity was clean. She had no past history of tuberculosis and was adequately vaccinated with BCG. A chest radiograph was obtained and found to be clear with nothing to suggest a primary focus of tuberculosis in the lungs. The patient was found to be non-reactive for HIV serology. We started by taking a biopsy of the lesion, which on histopathological examination showed non caseating epitheloid cell granulomas suggestive of tuberculosis. Infection with mycobacterium tuberculosis was further confirmed by PCR studies of the specimen as well. There was no evidence of malignancy (Figure 1).

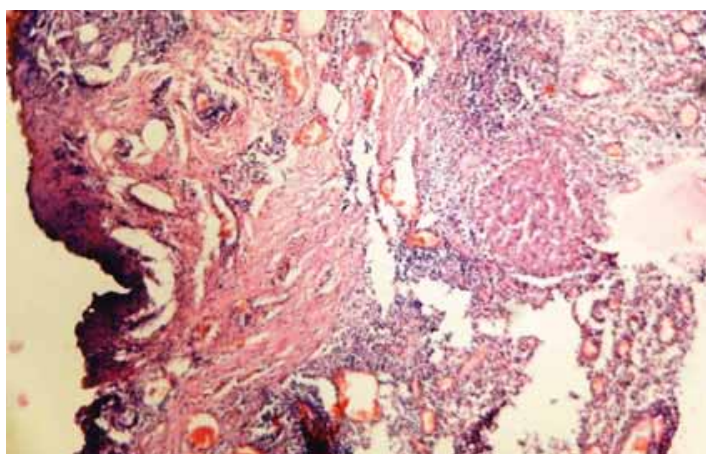

Figure 1(a) Haematoxylin and Eosin stained histopathological section of lesion biopsy at 10X

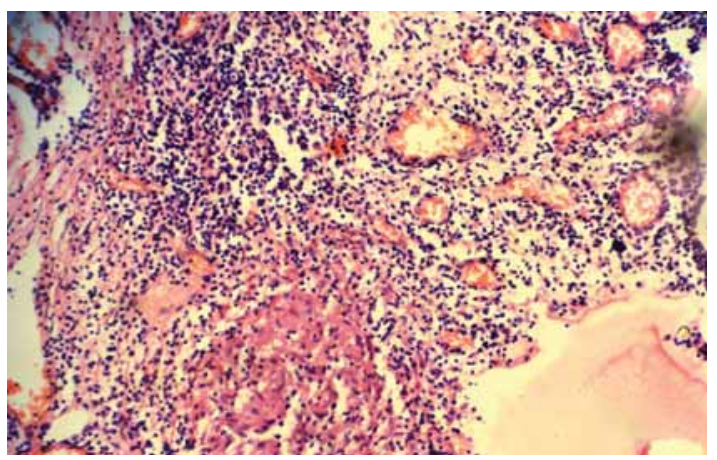

Figure 1(b) Magnifications respectively, showing non caseating epitheloid cell granulomas under light microscopy at $40 \mathrm{X}$
She was subsequently put on anti-tubercular therapy (ATT) with isoniazid, rifampicin, pyrazinamide and ethambutol (HRZE) for 3 months. By the end of 2 months of starting ATT the lesion had reduced in size and the patient was improving symptomatically. She later received a continuation therapy with isoniazid and rifampicin for 6 months. On subsequent follow up the lesion completely resolved and the patient is currently under follow up.

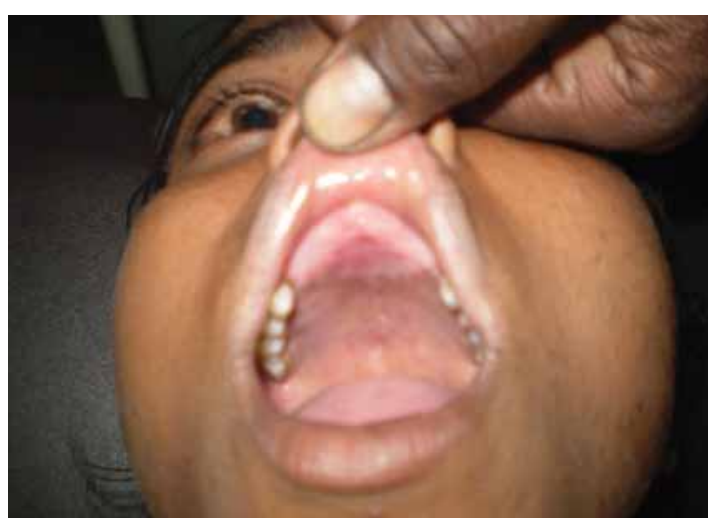

Figure 2 (a) before treatment

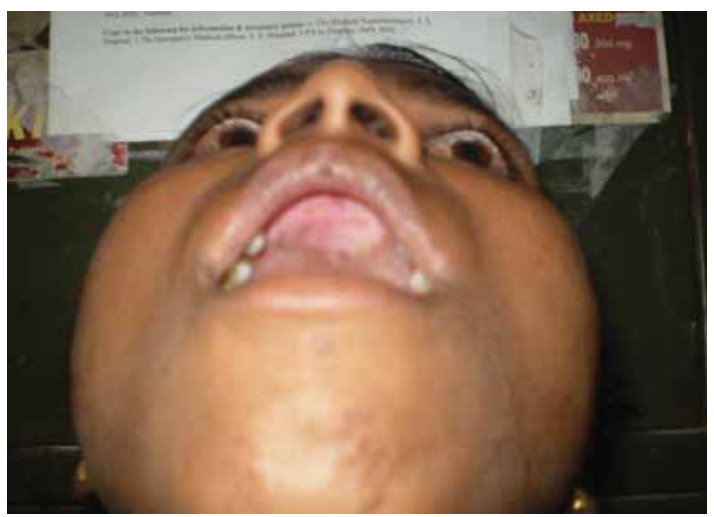

Figure 2 (母) after treatment

\section{DISCUSSION}

Although oral tuberculosis has been well documented with an incidence of accounting to $0.1-5 \%$ of all tuberculosis cases, ${ }^{2}$ tuberculous lesions of the upper aerodigestive tract have become rare. ${ }^{5}$ Tuberculous lesions of the oral cavity may be either primary, or secondary to disease elsewhere in the body, with secondary lesions being more common. ${ }^{6}$ Oral tuberculosis most commonly presents as ulcerations, typically as a stellate ulcer on the dorsum of the tongue with undermined edges, sloughed or granulated floor and indurated base. However, it can also present as swelling, nodules, discharge, fistulas or even 
diffuse inflammation of the oral cavity. ${ }^{2}$ Although the tongue is the commonest site for oral tuberculous lesions, they may also affect the mandible, gingiva, buccal mucosa, floor of mouth, lips and palate.,7-71 The hard palate is the rarest of all sites of oral involvement and was cited as the main or initial site of presentation of the oral TB lesion in $5 \%$ of the case reports ${ }^{12}$ according to a recent systematic review with $50 \%$ of the cases having evidence of a primary pulmonary pathology. ${ }^{2}$ Thus the present case report is one of the rarest presentations of tuberculosis.

The differential diagnosis of a tuberculous ulcer of the oral cavity includes aphthous ulcers, traumatic ulcers, syphilitic ulcers and malignancy. ${ }^{5}$ It is most likely that tuberculosis is only considered when the histological specimen reveals a granulomatous lesion. The diagnosis of tuberculosis is confirmed by the presence of acid-fast bacilli in the specimen, or more likely by culture of tubercle bacilli. Since oral tuberculosis is almost always secondary to pulmonary tuberculosis, sputum culture must also be carried out. Radiographic evidence of tuberculosis must also be sought. According to a recent review of the reported cases of oral tuberculosis symptoms had been present for an average of 6 months before diagnosis, $30 \%$ of these received antibiotic and/or antifungal therapy, $15 \%$ received corticosteroids or analgesic/antiinflammatory drugs while most of these cases were labelled as non-healing or unresponsive to treatment. ${ }^{2}$ The picture is very similar to the case presented here, which was previously misdiagnosed and treated inappropriately with antibiotics before we established the diagnosis of tuberculosis. This clearly emphasizes the importance of considering tuberculosis as a differential diagnosis in chronic oral lesions as well as calls for better co-ordination between dentists and physicians so as to ensure early diagnosis and prompt treatment of this disorder especially since it is an extremely treatable condition with cure rates approaching $85 \%{ }^{2}$

\section{REFERENCES}

1. Global tuberculosis control: WHO Report 2010. World Health Organization, Geneva, Switzerland, 2010.

2. Kakisi OK, Kechagia AS, Kakisis IK, Rafailidis $\mathrm{PI}$, Falagas ME. Tuberculosis of the oral cavity: a systematic review. Eur J Oral Sci 2010;118:103-9.

3. Snider DE, Castro KG. The global threat of drug-resistant tuberculosis. New England J Med 1998;338:1689-90.

4. Miziara ID. Tuberculosis affecting the oral cavity in Brazilian HIV-infected patients. Oral Surg Oral Med Oral Pathol Oral Radiol Endod 2005;100:179-82.

5. Von Arx DP, Husain A. Oral tuberculosis. British Dental Journal 2001;190:420-22.

6. Eng $\mathrm{H} L$, Lu S Y, Yang $\mathrm{C} \mathrm{H}$, Chen $\mathrm{W}$ J. Oral tuberculosis. Oral Surgery, Oral Medicine, Oral Pathology, Oral Radiology, \& Endodontics 1996;81:415-20.

7. Karthikeyan BV, Pradeep AR, Sharma CGD. Primary tuberculous gingival enlargement: a rare entity. J Can Dent Assoc 2006;72:645-48.

8. Rodrigues G, Carnelio S, Valliathan M. Primary isolated gingival tuberculosis. Braz $\mathrm{J}$ Infect Dis 2007;11:172-73.

9. Kavala M, Sudogan S, Can B, Sarigul S. Granulomatous cheilitis resulting from a tuberculide. Int J Dermatol 2004;43: 524-27.

10. Jones RF. Tuberculosis of the palate-an unusual presentation. J Laryngol Otol 1960;74:174-77.

11. Dinkar AD, Prabhudessai V. Primary tuberculous osteomyelitis of the mandible: a case report. Dentomaxillofac Radiol 2008;37:415-20.

12. Fujimoto T, Morishima T, Ueda M. Probable BCG osteomyelitis of the hard palate a case report. Int $\mathrm{J}$ Oral Maxillofac Surg 1996;25:145-46. 